\title{
Blind Equalization of IIR Channels using Hidden Markov Models
}

\author{
Vikram Krishnamurthy, Subhrakanti Dey, James P LeBlanc
}

Cooperative Research Center for Robust and Adaptive Systems, Department of Systems Engineering, Research School of Physical Sciences and Engineering, Australian National University, Canberra ACT 0200, Australia

Abstract - A computationally inexpensive suboptimal blind equalization algorithm is presented for noisy IIR channels. It is based on combining a recursive Hidden Markov Model (HMM) estimator with a relaxed SPR (strictly positive real) Extended Least Squares (ELS) scheme. Simulation studies show that the algorithm yields satisfactory results.

\section{Signal Model}

The abservations $y_{k}, k=1,2, \ldots, T$ are obtained as

$$
y_{k}=\frac{s_{k}}{C\left(z^{-1}\right)}+w_{k}, \quad w_{k} \sim N\left(0, \sigma_{w}^{2}\right)
$$

where $w_{k}$ is zero mean white Gaussian noise (WGN) with variance $\sigma_{w}^{2}$

$C\left(z^{-1}\right)=1-\sum_{i=i_{i}}^{P} c^{-i}$ (where $z^{-1}$ is the delay operator) denotes the unknown IIR channel. We assume that $C\left(x^{-1}\right)$ is stable, i.e., it has all its zeros outside the unit circle. $s_{k}$ denotes a $N$-state discrete-time homogeneous first-order Markov chain. Consequently, the state $s_{k}$ at time $k$ is one of $N$ known state levels $q=\left(\begin{array}{lll}q_{1} & q_{2} \ldots q_{N}\end{array}\right)^{\prime}$. The transition probability matrix is $A=\left(a_{i j}\right)$ where $a_{i j}=P\left(s_{t+1}=q_{j} \mid s_{t}=\right.$ $\left.q_{i}\right)$. Of course $a_{i j} \geq 0, \sum_{j=1}^{N} a_{i j}=1$, for each $i$. We assume that $s_{k}$ is irreducible.

\section{Algorithm Description}

Our algorithm is termed the HMM-ELS Blind Equalization Algorithm. It combines a relaxed SPR ELS scheme [2] and recursive $\mathrm{HMM}$ estimator [1] resulting in a suboptimal computationally efficient recursive (on-line) scheme [3]

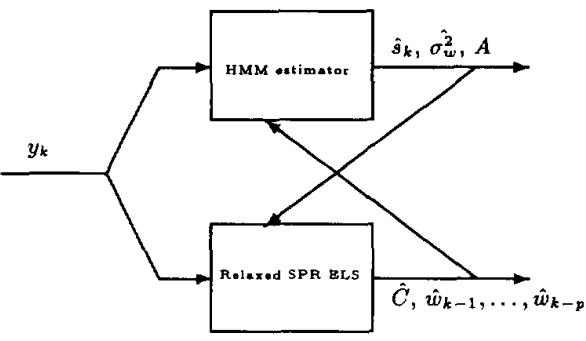

Figure 1: HMM-ELS Blind Equalization Algorithm

As shown in Fig.1, the HMM-ELS algorithm combines these two steps as follows:

1. At time $k$, the recursive HMM estimator yields estimate of the state of $s_{k}$, noise variance $\sigma_{w}^{2}$ and transition probabilities A.

2. The relaxed SPR ELS estimator gives on-line estimates of the channel parameters $c_{i}$ and $w_{k-i}, i \in\{1,2, \ldots, p\}$, denoted by $\hat{c}_{i}^{(k)}$ and $\hat{w}_{k-i}$ respectively.

\section{Simulation Studies}

Extensive simulation studies show that HMM-ELS yields excellent estimates even in low SNR [3]. It has been also shown in [3] that HMM-ELS performs better than Constant Modulus Algorithm (CMA). Here, we consider a jump time varying IIR(4) channel with coefficients

$$
C=\left\{\begin{array}{cc}
(-0.5-0.40 .30 .2)^{\prime} & 1 \leq k \leq 10000 \\
(1.0-0.90 .7-0.36)^{\prime} & 10000<k \leq 50000
\end{array}\right.
$$

The input the channel is a two state markov chain with $a_{11}=$ $a_{22}=0.9, q_{1}=-q_{2}=1$. Also $\sigma_{w}=0.6$. Figure 2 shows how the HMM-ELS algorithm tracks the channel coefficients with a forgetting factor of $\lambda=0.995$.

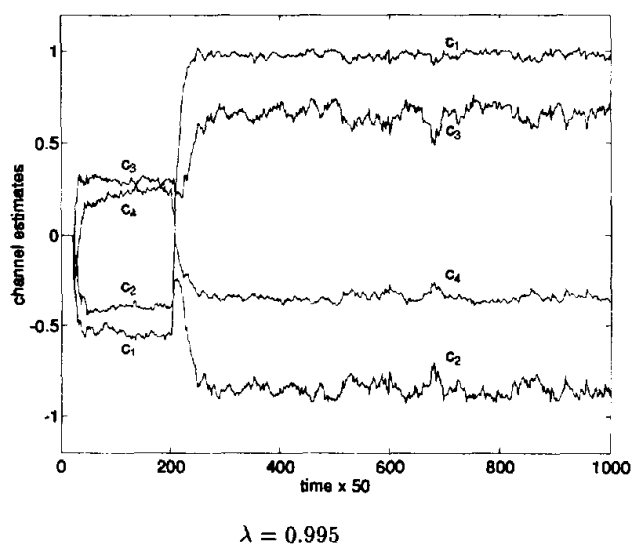

Figure 2: Equalization of "jump" time-varying channel

\section{RefERENCES}

[1] V. Krishnamurthy, J.B. Moore, On-line Estimation of Hidlen Markov Model Parameters based on the Kullback-Leibler Information Measure, IEEE Trans on Signal Processing, Vol. 41, No. 8, pp. 2557-2573, August, 1993.

[2] J.B. Moore, et.al, Identication/Prediction algorithms for $A R$ MAX models with relaxed positive real conditions, Int. Journal of Adaptive Control and Signal Processing, pp.49-67, 1990.

[3] V. Krishnamurthy, S. Dey and J P LeBlanc, Blind Equlisation of IIR Channels using Hidden Markov Models and Extended Lecst Squares, submitted to IEEE Trans on Signal Processing. 